\title{
A Viewpoint on the Information Sharing Paradox
}

\author{
Shane P. Stenner ${ }^{1}$ William Rice ${ }^{2}$ Scott D. Nelson ${ }^{1}$ \\ ${ }^{1}$ Department of Biomedical Informatics, Vanderbilt University \\ Medical Center, Nashville, Tennessee, United States
}

2 Vanderbilt Health Affiliated Network, Nashville, Tennessee, United States

Appl Clin Inform 2020;11:460-463.

\section{Introduction}

Electronic health records (EHRs) have decreased barriers to data capture, ${ }^{1}$ overwhelming clinicians with information, paradoxically leading to decreased efficiency for many providers and often making retrieval of clinically pertinent data more difficult. ${ }^{2}$ Now, a new ruling aims to increase data sharing, potentially worsening information overload and implicating health care providers and organizations of "information blocking."

\section{The 21st Century Cures Act and Information Blocking}

In 2016, the 21st Century Cures Act (Cures Act) was signed into law, ${ }^{3}$ introducing provisions promoting cancer research, precision medicine, and expediting the approval process for new drugs and devices. It also included provisions designed to maximize the potential of Health Information Technology (Health IT) by supporting the access, exchange, and use of electronic health information (EHI), and penalizing intentional limits on EHI sharing (information blocking) by health care providers, certified health IT, exchanges, or networks. The Cures Act defined "information blocking" broadly, addressed activities that unreasonably limit the availability and use of EHI, and charged the U.S. Department of Health and Human Services (HHS) to identify "reasonable and necessary" activities that do not constitute information blocking (i.e., exceptions). In 2019, HHS published a proposed rule, which was recently finalized, defining these expectations and authorized HHS with investigatory and enforcement authority over information blocking, including the ability to issue substantial monetary penalties for violations. $^{4}$

Increased information sharing as a result of the finalized rule will add to the deluge of data and information overload already affecting individual providers and organizations. 5,6 More than having too much data, information overload occurs when more information is presented than can be
Address for correspondence Shane P. Stenner, MD, MS, Department of Biomedical Informatics, Vanderbilt University Medical Center, 3401 West End Avenue, Suite 630, \#644, Nashville, TN 37203, United States (e-mail: shane.stenner@vumc.org).

readily processed, thereby exceeding coping strategies, causing a loss of situational awareness, increasing the risk of missing important information, increasing times finding and organizing information, and potentially leading to physician burnout. ${ }^{7-11}$ More information does not necessarily mean more effective decision making or care..$^{8,12}$

\section{Impact on Information Receivers}

The finalized rule can be expected to have a significant impact on existing information sharing arrangements by enforcing interoperability and information sharing for all health care providers and organizations. The authors fully support freely sharing EHI; however, the finalized rule fails to directly address the needs of and impact on information receivers (i.e., health care entities and providers). In environments of low (or no) cost information transmission (the incremental cost of sending large amounts of data being low, or even resulting in low quality or accuracy), informationsenders can take advantage of the technology for their benefit, overwhelming information-receivers. For example, the effort to send an alert or notification to a large group of clinicians is small compared with the amount of effort expended in reading and responding to that alert or notification (especially if chart review is needed). In turn, the information-receiver may start filtering, summarizing, and routing information, potentially breaking the information blocking provision.

Providers should be expected to know all relevant information about a patient that is necessary to deliver appropriate care. However, easily distinguishing relevant information from nonrelevant information is not possible in our current systems. If one considers only existing data in a single health care entities' modern EHR, it is already impossible for the human brain to synthesize all information for a typical patient. Health IT solutions to address the challenges of information overload are important and should be pursued. However, immediately requiring receipt and reconciliation (c) 2020 Georg Thieme Verlag KG
Stuttgart · New York
DOI https://doi.org/

10.1055/s-0040-1713413. ISSN 1869-0327. 
of all information without consideration of how that effort will actually be done by real, practicing clinicians is unrealistic and, in the authors' opinion, likely to fail. The deluge of data and information from well-intended actors has already begun, and clinician efforts to reduce information overload by filtering could paradoxically obscure critical information and harm patients.

SPAM email and "robocalls" to personal mobile phones provide everyday examples of this unequal information-power relationship (sender vs. receiver), where an information sender can quickly and easily send useless information to a large number of people. While other technology leaders have found ways to separate the "signal" from the "noise" (i.e., automatic email prioritization, sorting, and SPAM filters), modern EHR vendors provide limited tools to filter, block, or prioritize external information. With the finalized rules, it remains unclear if EHR vendors or clinical entities (information-receivers) will be subject to information blocking penalties for attempting to filter information deemed less clinically relevant. Health care entities have a variety of competing incentives relative to their information and clinical data are notoriously open to differing opinions and interpretations.

One such example is the use of the Direct Messaging standard. Direct is a secure, email-like method for exchanging patient information. The Direct standard puts some safety mechanisms in place to validate information senders but there is no governance over what constitutes clinically useful or desired information for the information receiver. Recently, a provider complained to the authors about the high number of "Patient Safety and Health Consideration" messages he received via Direct from a large national pharmacy benefits manager and the poor clinical relevance of those "considerations." The provider's opinion was that the "considerations" appeared to be made in isolation of the patient's overall condition and health history, offered little added value, and appeared to be generated from billing data. For most of the "considerations," the provider reported spending 10 to 15 minutes reviewing and documenting why the safety and health consideration did not apply to their patient's specific case.

This provider's account may sound familiar. It follows a similar pattern of our collective experiences with other wellintended information "pushes" into the clinical workflow. In recent years clinicians have been overwhelmed with "alert fatigue" from nonspecific medication alerts and other interruptive clinical decision support. ${ }^{13,14}$ Yet, we continue to increase data following into clinical systems, such as genomic data and patient generated data from wearables and connected devices, to clinicians who are overwhelmed, unprepared, or unwilling to use them. ${ }^{15-19}$ Additionally, clinicians (or their staff) are now spending more and more time reconciling outside information, an activity viewed by many as an uncompensated administrative burden with minimal clinical impact, ${ }^{20}$ yet required by Merit-based Incentive Payment System quality measures. EHI receivers will have different opinions, preferences, and thresholds for receiving such external information, which may vary by institution, specialty, clinic, or even the individual. The policies, regulations, and technology need to reflect and support these variations.

\section{Considerations for the Future of Information Sharing}

Health care organizations and clinicians already struggle with information overload. Data and information may be received in several ways, including integrated electronic delivery, physical devices (i.e., CDs), and printed/faxed documents. ${ }^{21,22}$ Many of the data received today are not integrated, ignored, or otherwise filtered in rudimentary ways. The resource costs are high for handling, filtering, labeling, and configuring these data in an attempt to file only clinically relevant information into EHRs. We cannot assume that as electronic delivery of information increases, resource costs of receiving information will necessarily decrease; given the ease of sending information and the potential benefits to some health information "senders," we can likely expect the resource costs for health information-receiving entities to increase. Every message creates additional burden on the receiver, but only some messages improve patient care.

Therefore, every health care entity should consider if they are:

1. Prepared to filter and make use of increased data liquidity.

2. Able to protect patients by ensuring important signals are "caught."

3. Able to protect clinicians from information overload and undue administrative burden.

4. At an increased risk of the information blocking provision through pursuit of any of the above aims.

Potential future solutions to the increasing influx of information include systematic changes in clinical workflows, increasing responsibilities of nonprovider members of the health care team, and EHR functional enhancements. However, none of these solutions are mandated or particularly supported by the Cures Act and some may require additional law and policy changes to address appropriate scope of practice questions. The regulation amplifies the burden of information reconciliation on information-receiving health care entities (not just providers), without any incentives or protections for information receivers to process and reconcile information well. While systematic changes and informatics solutions may eventually be helpful, they require additional funding, resources, and time while health care systems, in general, are functioning on decreasing margins. Historically, the provider is ultimately responsible for the patient's care and without significant changes to tools, policies, and workflows, the only person with appropriate knowledge and clinical responsibility to reconcile most clinical information will be the provider. Policy makers and EHR vendors must consider the implications of the information blocking rules on information-receiving entities. In particular, the authors recommend:

1. Safe harbors for routing, filtering, blocking, or unsubscribing from information.

2. Standards and electronic tools (EHRs and health information service providers) necessary for routing, filtering, synthesizing, summarizing, blocking, and unsubscribing. 
3. Public policies and tools that allow customization/personalization for data and information opt-out at a variety of organizational or individual levels.

4. Safeguards for clinicians and organizations against bad actors, which could use these interfaces and direct contact to physicians for malicious or unethical purposes.

\section{Conclusion}

Increased data sharing is intended to improve health care, but information overload can also decrease the quality and efficiency of care-thus the information sharing paradox. While clinicians (and their IT systems) must be increasingly open to engage in all forms of information sharing, policies and technologies must advance rapidly to protect information-receiving entities. Without considering the diminishing clinical utility of information, increasing burden of information overload, and the needs of information-receiving entities to route, filter, synthesize, and summarize data and to block or unsubscribe from information-senders, the health care system risks significant harm to patients and providers alike.

\section{Clinical Relevance Statement}

More information does not necessarily mean more effective patient care. Overwhelming clinicians with information can paradoxically lead to decreased efficiency for many providers. Health IT systems are increasingly engaging in all forms of information sharing. At the same time, there should be policies and technologies to help or protect clinicians and organizations process the deluge of information.

\section{Multiple Choice Questions}

1. The information blocking ruling is an executive order of? a. Affordable Care Act (ACA).

b. 21st Century Cures Act.

c. American Recovery and Reinvestment Act/Health Information Technology for Economic and Clinical Health Act.

d. None of the above.

Correct Answer: The correct answer is option b. The information blocking ruling is an executive order of the $21^{\text {st }}$ Century Cures Act.

2. Information blocking applies to which of the following entities?
a. Health care providers.
b. Health IT developers of certified health IT.
c. Health information exchanges.
d. Health information networks.
e. All of the Above.

Correct Answer: The correct answer is option e. Information blocking applies to all of the listed entities.

3. Information overload is associated with which of the following effects?

a. Loss of situational awareness.
b. Improved decision-making ability.
c. Increased productivity.
d. None of the above.

Correct Answer: The correct answer is option a. Loss of situational awareness is directly associated with information overload.

4. Which of the following are methods for sharing patient information?
a. Compact disks.
b. Faxes.
c. DIRECT messages.
d. Health information exchange integration.
e. All of the above.

Correct Answer: The correct answer is option e. Patient information may be appropriately shared through all four of the listed methods.

5. Policy makers and EHR vendors are recommended to consider which of the following implications of the information blocking rules on information-receiving entities?

a. Safe harbors for routing, filtering, blocking, or unsubscribing from information.

b. Standards and electronic tools (EHRs and HISPs) necessary for routing, filtering, synthesizing, summarizing, blocking, and unsubscribing.

c. Policies and tools that allow customization/personalization for data and information opt-out at a variety of organizational or individual levels.

d. Safeguards for clinicians and organizations against bad actors, which could use these interfaces and direct contact to physicians for malicious or unethical purposes.

e. All of the above.

Correct Answer: The correct answer is option e. The authors describe four recommendations to policy makers and EHR vendors regarding the implications of the information blocking rules.

\section{Protection of Human and Animal Subjects}

This manuscript does not present research data for which human subjects approval is required.

Funding

None.

\section{Conflict of Interest}

None declared.

\section{Acknowledgments}

The authors would like to acknowledge Mark Frisse, MD, MS, MBA, FACMI, for his thoughtful and critical review of the manuscript.

\section{References}

1 Health IT. gov. Capturing high quality electronic health records data to support performance improvement: ONC; 2013. Available at: https://www.healthit.gov/sites/default/files/onc-beacon-lg3-ehrdata-quality-and-perform-impvt.pdf. Accessed February 18, 2020 
2 Nelson SD, LaFleur J, Del Fiol G, Evans RS, Weir CR. Reading and writing: qualitative analysis of pharmacists' use of the EHR when preparing for team rounds. AMIA Annu Symp Proc 2015; 2015:943-952

3 21st Century Cures Act, Public Law No: 114-255; 2016. Available at: https://www.congress.gov/bill/114th-congress/house-bill/34/ text. Accessed August 20, 2019

4 Federal Register Volume 84, Issue 42 (March 4, 2019). Office of the Federal Register, National Archives and Records Administration; 2019:7261-7791

5 McDonald CJ. Toward electronic medical record alerts that consume less physician time. JAMA Intern Med 2013;173(18): $1755-1756$

6 Singh H, Sittig DF. Toward electronic medical record alerts that consume less physician time-reply. JAMA Intern Med 2013;173 (18): 1756

7 Miller JG. Living Systems. New York: McGraw-Hill Co; 1978

8 Heuer RJ. Psychology of Intelligence Analysis. Washington, D.C.: Center for the Study of Intelligence, Central Intelligence Agency;1999

9 Tai-Seale M, Dillon EC, Yang Y, et al. Physicians' well-being linked to in-basket messages generated by algorithms in electronic health records. Health Aff (Millwood) 2019;38(07):1073-1078

10 Singh H, Spitzmueller C, Petersen NJ, Sawhney MK, Sittig DF. Information overload and missed test results in electronic health record-based settings. JAMA Intern Med 2013;173(08):702-704

11 Gardner RL, Cooper E, Haskell J, et al. Physician stress and burnout: the impact of health information technology. J Am Med Inform Assoc 2019;26(02):106-114

12 Leetaru K. Does More Data Really Lead To Better Decision Making? Forbes; 2016. Available at: https://www.forbes.com/sites/kalevleetaru/2016/06/14/does-more-data-really-lead-to-better-decision-making/\#43728e121895. Accessed February 18, 2020
13 Payne TH. EHR-related alert fatigue: minimal progress to date, but much more can be done. BMJ Qual Saf 2019;28(01):1-2

14 Gregory ME, Russo E, Singh H. Electronic health record alertrelated workload as a predictor of burnout in primary care providers. Appl Clin Inform 2017;8(03):686-697

15 Najafzadeh M, Davis JC, Joshi P, Marra C. Barriers for integrating personalized medicine into clinical practice: a qualitative analysis. Am J Med Genet A 2013;161A(04):758-763

16 Arar N, Seo J, Abboud HE, Parchman M, Noel P. Providers' behavioral beliefs regarding the delivery of genomic medicine at the Veterans Health Administration. Per Med 2010;7(05):485-494

17 Selkirk CG, Weissman SM, Anderson A, Hulick PJ. Physicians' preparedness for integration of genomic and pharmacogenetic testing into practice within a major healthcare system. Genet Test Mol Biomarkers 2013;17(03):219-225

18 Zhang R, Burgess ER, Reddy MC, et al. Provider perspectives on the integration of patient-reported outcomes in an electronic health record. JAMIA Open 2019;2(01):73-80

19 Baig MM, GholamHosseini H, Moqeem AA, Mirza F, Lindén M. A systematic review of wearable patient monitoring systems current challenges and opportunities for clinical adoption. J Med Syst 2017;41(07):115

20 Rao SK, Kimball AB, Lehrhoff SR, et al. The impact of administrative burden on academic physicians: results of a hospital-wide physician survey. Acad Med 2017;92(02):237-243

21 Hughes CA, Allen P, Bentley M. eReferrals: why are we still faxing? Aus J Gen Pract 2018;47(1/2):51

22 Farr C. 'Ditch the Disk' group of tech execs is spurring the medical industry to move on from CD-ROMs: CNBC; 2020 [updated 2/16/ 2020]. Available at: https://www.cnbc.com/2020/02/14/salesforce-cmo-ashwini-zenooz-urges-healthcare-to-move-on-fromcd-roms.html. Accessed February 18, 2020 